MATEC Web of Conferences 22,03015 (2015)

DOI: $10.1051 /$ matec conf/ 20152203015

(C) Owned by the authors, published by EDP Sciences, 2015

\title{
Application of Strain Energy on BIW Mode Optimization
}

\author{
Guangbao Chang* \\ SAIC GM Wuling Automobile Co., Ltd. Liuzhou, Guangxi, China \\ Huifang Zhao \\ Guangxi Liugong Machinery Co.,Ltd ., Liuzhou, Guangxi, China \\ Jingqiang Liang, Juncheng Lv, Shuyang Li \& Xiaobo Ni \\ SAIC GM Wuling Automobile Co., Ltd. Liuzhou, Guangxi, China
}

\begin{abstract}
This paper takes the BIW model as the research object, completes modal analysis, and verifies the finite element model by comparing the simulation results with the test results. In order to improve the frequency of BIW, the weak structure of D pillar is found and then optimized by the method of strain energy, and the frequency of BIW is changed from $28.80 \mathrm{~Hz}$ to $32.15 \mathrm{~Hz}$. Finally, the method of strain energy has great positive effects on modal optimization.
\end{abstract}

Keywords: strain energy; mode; optimization

\section{INTRODUCTION}

At present, the automobile trade competition is fierce and upgrading fast, and it depends on the design of BIW (Body In White). In development of new cars, the design of BIW is dominant and its characteristic has influences on the performance of vehicle. In order to gain the good performance, it's required that the BIW should have high stiffness and good vibration performance, and those performances are gained by modal analysis of the finite element method and test ${ }^{[1-2]}$. As for the current optimization method, Hao Qu, Xiaohua Wang and Tong Zhang from Tongji University apply the modal optimization on a car's BIW by utilizing the DOE ${ }^{[3]}$. Changming Chen and Qiang Xiao apply the sensitivity method on modal analysis This paper establishes the finite element model of BIW and takes the modal analysis. The result is consistent with the test. Finally, it provides a new way, namely the strain energy method, to optimize the modal BIW.

\section{BASIC THEORY OF MODAL ANALYSIS}

A differential equation of multi-degree-of-freedom linear system is shown as follows:

$[\mathrm{M}]\{\ddot{\mathrm{x}}(\mathrm{t})\}+[\mathrm{C}]\{\dot{\mathrm{x}}(\mathrm{t})\}+[\mathrm{K}]\{\mathrm{x}(\mathrm{t})\}=\{\mathrm{F}(\mathrm{t})\}$

Where $[\mathrm{M}] 、[\mathrm{C}]$ and $[\mathrm{K}]$ are respectively the mass, damping and stiffness matrix. $\{\ddot{\mathrm{x}}(\mathrm{t})\},\{\dot{\mathrm{x}}(\mathrm{t})\}$ and $\{\mathrm{x}(\mathrm{t})\}$ are respectively the acceleration, velocity and displacement of node. $\{\mathrm{F}(\mathrm{t})\}$ is the exciting force.

The equation is described by the physical coordinate. When the dofs (degree of freedoms) are large, it becomes very hard to solve. However, the modal

*Corresponding author: cgb19840815@163.com analysis method uses the modal coordinate to replace the physical coordinate and that turns the coupled equation into many independent coordinate equations, so the modal parameters are easily solved. With no damping and force, the equation is changed into:

$[\mathrm{M}]\{\ddot{\mathrm{x}}(\mathrm{t})\}+[\mathrm{K}]\{\mathrm{x}(\mathrm{t})\}=0$

After transformed, the characteristic equation is shown as follows:

$\left|\mathrm{K}-\omega^{2} \mathrm{M}\right|=0$

If the system has $\mathrm{n}$ dofs, then it exits $\mathrm{n}$ characteristics. Modal analysis is a process of solving the characteristic root $\omega_{\mathrm{i}}(\mathrm{i}=1,2, \cdots, \mathrm{n})$, and these characteristic roots represent the natural frequencies or resonant frequencies. Eigenvector corresponding to the natural frequency is called the orthogonal mode or the mode shape. When the structure vibrates, it's the linear combination of orthogonal mode at any time. One property of the system is termed as the circular natural frequency of the structure. The subscript indicates the "natural" for the SDOF system. In systems having more than one mass degree of freedom and more than one natural frequency, the subscript may indicate a frequency number. For an SDOF system, the circular natural frequency is given as follows

$\omega_{\mathrm{n}}=\sqrt{\mathrm{k} / \mathrm{m}}$

The circular natural frequency is specified in units of radians per unit time.

The natural frequency is defined as follows:

$\mathrm{f}_{\mathrm{n}}=\omega_{\mathrm{n}} / 2 \pi$

The natural frequency is often specified in terms of cycles per unit time, commonly cycles per second(cps), which is more commonly known as Hertz $(\mathrm{Hz})$. The modal frequency response analysis is an alternate approach to compute the frequency response of a 


\section{MATEC Web of Conferences}

structure. This method uses the mode shapes of the structure to reduce the size, uncouple the equationsof motion (when modal or no damping is used), and make the numerical solution more efficient. Since the mode shapes are typically computed as part of the characterization of the structure, the modal frequency response is a natural extension of a normal modes analysis.

As a first step in the formulation, transform the variables from physical coordinates $\mathrm{s}\{\mathrm{u}(\omega)\}$ to modal coordinate $\{\xi(\omega)\}$ by assuming:

$$
\{\mathrm{x}\}=[\Phi]\{\xi(\omega)\} \mathrm{e}^{\mathrm{i} \omega \mathrm{t}}
$$

The mode shapes [Ф]are used to transform the problem in terms of the behavior of the modes as opposed to the behavior of the grid points. Equation (6) represents an equality if all modes are used; however, because all modes are rarely used, the equation usually represents an approximation. Proceed and temporarily ignore all damping, which results in the un-damped equation for harmonic motion at forcing frequency:

$-\omega^{2}[\mathrm{M}]\{\mathrm{x}\}+[\mathrm{K}]\{\mathrm{x}\}=\{\mathrm{p}(\omega)\}$

Substitute the modal coordinates in Equation (6) for the physical coordinates in Equation (7) and divide them by $\mathrm{e}^{\mathrm{i} \omega \mathrm{t}}$, it is obtained as follows:

$-\omega^{2}[\mathrm{M}][\Phi]\{\xi(\omega)\}+[\mathrm{K}][\Phi]\{\xi(\omega)\}=\{\mathrm{p}(\omega)\}$

Now this is the equation of motion in terms of the modal coordinates. At this point, however, the equations remain coupled.

To uncouple the equations, pre-multiply by $[\Phi]^{\mathrm{T}}$, it is obtained as follows:

$-\omega^{2}[\Phi]^{\mathrm{T}}[\mathrm{M}][\Phi]\{\xi(\omega)\}$

$+[\Phi]^{\mathrm{T}}[\mathrm{K}][\Phi]\{\xi(\omega)\}=[\Phi]^{\mathrm{T}}\{\mathrm{p}(\omega)\}$

Where:

$[\Phi]^{\mathrm{T}}[\mathrm{M}][\Phi]=$ modal (generalized) mass matrix

$[\Phi]^{\mathrm{T}}[\mathrm{K}][\Phi]=$ modal (generalized) stiffness matrix

$[\Phi]^{\mathrm{T}}\{\mathrm{p}(\omega)\}=$ modal force vector

The final step uses the orthogonality property of the mode shapes to formulate the equation of motion in terms of the generalized mass and stiffness matrices, which are diagonal matrices. These diagonal matrices do not have the off-diagonal terms that couple the equations of motion. Therefore, in this form, the modal equations of motion are uncoupled. And in this uncoupled form, the equations of motion are written as a set of uncoupled single degree-of-freedom systems as follows:

$-\omega^{2} \mathrm{~m}_{\mathrm{i}} \xi_{\mathrm{i}}(\omega)+\mathrm{k}_{\mathrm{i}} \xi(\omega)=\mathrm{p}_{\mathrm{i}}(\omega)$

Where:

$\mathrm{m}_{\mathrm{i}}=\mathrm{i}$-th modal mass

$\mathrm{k}_{\mathrm{i}}=\mathrm{i}$-th modal stiffness

$\mathrm{p}_{\mathrm{i}}=\mathrm{i}$-th modal force

The modal form of the frequency response equation of motion is much faster to solve than solving by the direct method because it is a series of uncoupled single degree-of-freedom systems. Once the individual modal responses $\xi_{\mathrm{i}}(\omega)$ are computed, physical re- sponses are recovered as the summation of the modal responses using

$$
\{\mathrm{x}\}=[\Phi]\{\xi(\omega)\} \mathrm{e}^{\mathrm{i} \omega \mathrm{t}}
$$

\section{BIW MODAL ANALYSIS}

\subsection{Finite Element Model}

The FEM is established based on the software of HyperMesh, the size of element is $10 \mathrm{~mm} * 10 \mathrm{~mm}$ and the key part is needed to remesh finely but the smallest size is not less than $3 \mathrm{~mm}$, the sum of shell elements is 639440 which the sum of quad4 elements is 609968 that over $95.4 \%$. It meets the requirement that the percentage of tria3 elements is less than $5 \%$. It used ACM to simulate the spot welding, and RBE2 to simulate the seam welding. The material parameters are shown as follows:

$[\mathrm{E}]=2.07+11 \mathrm{~Pa},[\mathrm{NU}]=0.3,[\mathrm{RHO}]=7.83 \mathrm{e}-9 \mathrm{ton} / \mathrm{mm}^{\wedge} 3[5-6]$.

\subsection{Simulation results of BIW Mode}

The main mode shapes and frequencies are shown in Table 1:

Table1. Mode shape and frequency

\begin{tabular}{|l|l|l|}
\hline & Shape & Frequency $(\mathrm{Hz})$ \\
\hline \multirow{3}{*}{ BIW Mode } & $1^{\text {st }}$ Tail torsion & 28.80 \\
\cline { 2 - 3 } & $1^{\text {st }}$ Vertical bending & 45.63 \\
\cline { 2 - 3 } & $1^{\text {st }}$ Whole body torsion & 41.73 \\
\hline
\end{tabular}

The simulation results are shown by Figure 1, Figure 2 and Figure 3:

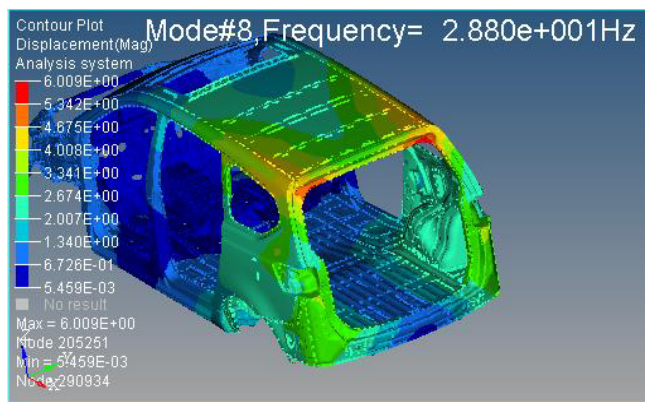

Figure 1. $1^{\text {st }}$ Tail torsion

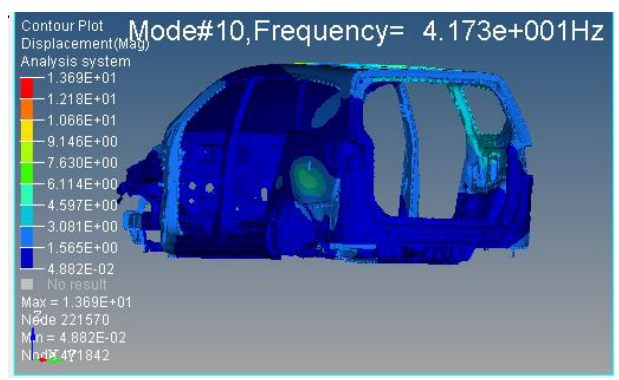

Figure 2. $1^{\text {st }}$ Vertical bending 
ICETA 2015

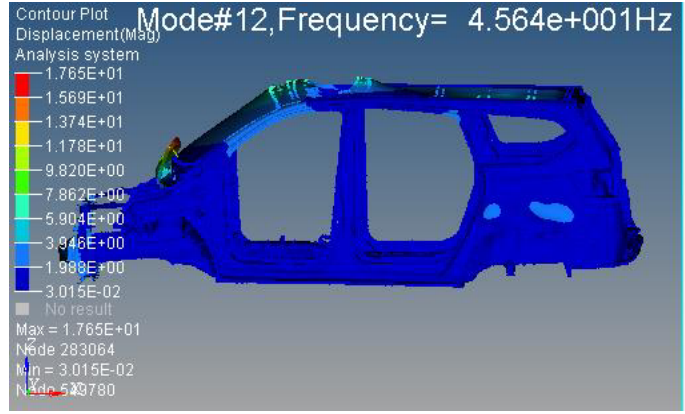

Figure 3. $1^{\text {st }}$ Whole body torsion

\subsection{Test results of BIW mode}

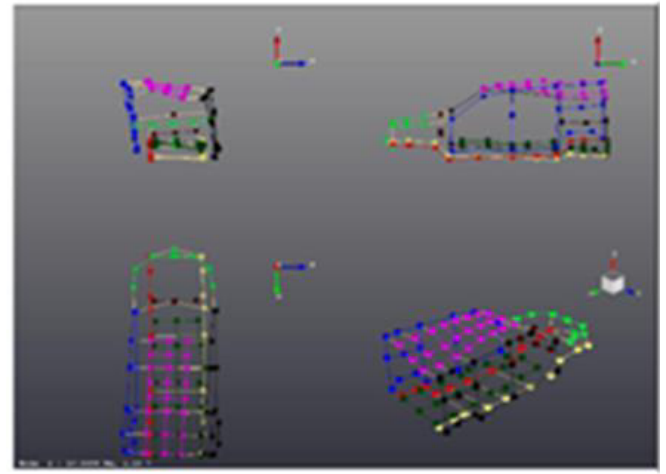

\begin{tabular}{|c|c|c|c|}
\hline $\begin{array}{c}\text { Mode } \\
\text { No. }\end{array}$ & $\begin{array}{c}\text { Frequency } \\
(\mathrm{Hz})\end{array}$ & Damping & $\begin{array}{c}\text { Mode Shape } \\
\text { Description }\end{array}$ \\
\hline Mode 1. & $28.89 \nu$ & $1.25 \%$ & Tail torsion \\
\hline
\end{tabular}

Figure 4. Test mode result of tail torsion

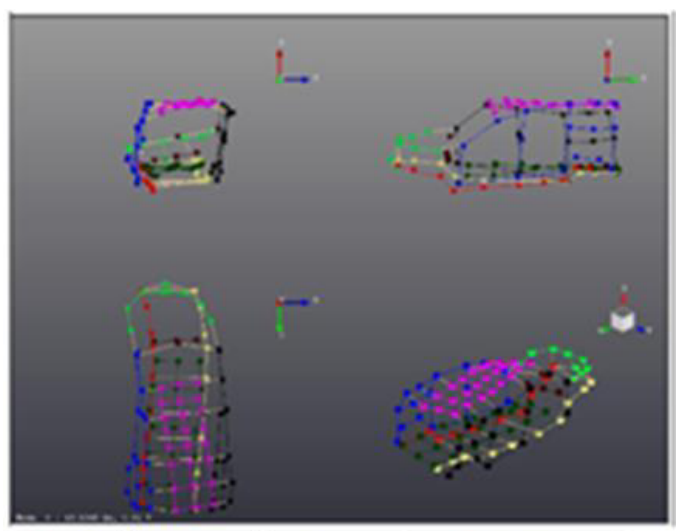

\begin{tabular}{|c|c|c|c|}
\hline $\begin{array}{c}\text { Mode } \\
\text { No. }\end{array}$ & $\begin{array}{c}\text { Frequency } \\
(\mathrm{Mz}) \nu\end{array}$ & Damping $\nu$ & $\begin{array}{c}\text { Mode Shape } \\
\text { Description }\end{array}$ \\
\hline Mode $5 \cdot$ & $43.61 \nu$ & $0.91 \%$ & Vertical bending $\nu$ \\
\hline
\end{tabular}

Figure 5. Test mode result of tail vertical bending

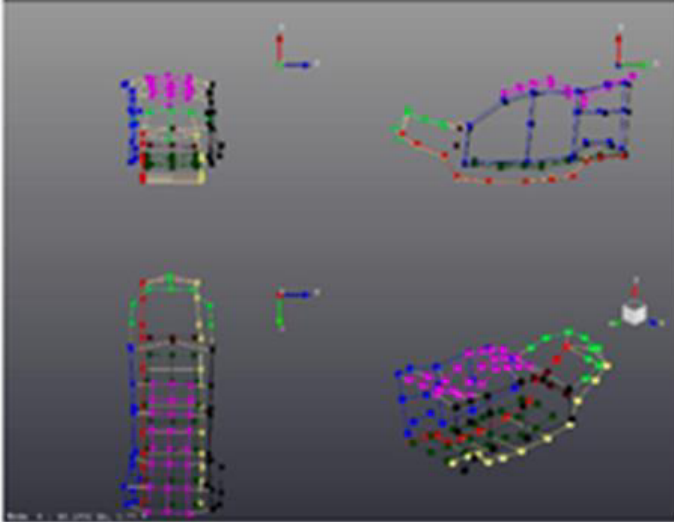

\begin{tabular}{|c|c|c|c|}
\hline $\begin{array}{c}\text { Mode } \\
\text { No. }\end{array}$ & $\begin{array}{c}\text { Frequency } \\
(\mathrm{Mz})\end{array}$ & Damping & $\begin{array}{c}\text { Mode Shape } \\
\text { Description }\end{array}$ \\
\hline Mode 6* & $46.19 \cdot$ & $0.77 \%$ & Whole body torsion \\
\hline
\end{tabular}

Figure 6. Test mode result of tail whole body torsion

The frequency of first tail torsion mode is $28.89 \mathrm{~Hz}$; $1^{\text {st }}$ whole body torsion is $43.61 \mathrm{~Hz} ; 1^{\text {st }}$ vertical bending is $46.19 \mathrm{~Hz}$. The test results are shown in Figure 4, Figure 5 and Figure 6.

\subsection{Results analysis}

Table 2.Comparison of the simulation with test results.

\begin{tabular}{|c|c|c|c|}
\hline \multirow{2}{*}{ Mode } & \multicolumn{2}{|c|}{ Frequency $(\mathrm{Hz})$} & consistency \\
\cline { 2 - 4 } & simulation & test & rate \\
\hline $1^{\text {st }}$ Tail torsion & 28.80 & 28.89 & $99.7 \%$ \\
\hline $1^{\text {st }}$ Vertical bending & 45.63 & 46.19 & $98.8 \%$ \\
\hline $1^{\text {st }}$ Whole body torsion & 41.73 & 43.61 & $98.12 \%$ \\
\hline
\end{tabular}

The consistency rates exceed $95 \%$ from the comparison results, and which explains that the FEM is right and believable, so the model can be used to further optimization work.

The frequency of first tail torsion mode is $28.89 \mathrm{~Hz}$, which does not meet the requirement of the performance goal. Those results in the lower stiffness at the tail position have severely influences on the NVH performance and the bad fatigue performance leading to the tail crack as well. Then, the strain energy method is used to optimize the BIW mode.

\section{OPTIMIZING THE BIW MODE}

\subsection{Basic theory}

The strain energy can be used to find the changing of 


\section{MATEC Web of Conferences}

part performance, and point out the exact position from document ${ }^{[7]}$. The mode $i$ of characteristic equation with no damping is shown as follows:

$[\mathrm{K}]\{\varnothing\}_{\mathrm{i}}=\omega_{\mathrm{i}}^{2}[\mathrm{M}]\{\varnothing\}_{\mathrm{i}}$

Where the left equation is the elasticity restorable force vector, and the right equation is the inertia force vector. According to the D'Alembert's principle, the elasticity deformation is caused by inertia force. It is expressed by elasticity restorable force as follows:

$[\mathrm{K}]\{\varnothing\}_{\mathrm{i}}=\sum_{\mathrm{k}=1}^{\mathrm{m}} \int_{\mathrm{V}_{\mathrm{k}}}[\mathrm{D}]\{\varphi\}_{\mathrm{i}_{\mathrm{k}}} \mathrm{dv}=\omega_{\mathrm{i}}^{2}[\mathrm{M}]\{\varnothing\}_{\mathrm{i}}$

Where $m$ is the sum of structure parts; $V_{k}$ is the $\mathrm{k}^{\text {th }}$ volume of structure parts; [D] is the constitutive matrix; $\{\varphi\}_{\mathrm{i}_{\mathrm{k}}}$ is the ith mode strain distribution of the kth structure. $\{\varphi\}_{\mathrm{i}}$ is generated by inertia force from mode decomposition, which is the strain mode corresponding to the displacement mode. The displacement and strain mode are both ratios, and they have the same frequency at the same mode.

In the process of mode analysis, the strain energy distribution is easily gained by the software of Nastran ${ }^{[8]}$.The large elastic displacement is found by strain energy distribution, so the weakness part is optimized.

\subsection{Strain energy analysis of $1^{\text {st }}$ Tail torsion}

The strain energy result is attained from mode result, and the strain energy distribution of $1^{\text {st }}$ tail torsion is shown in Figure 7 as follows:

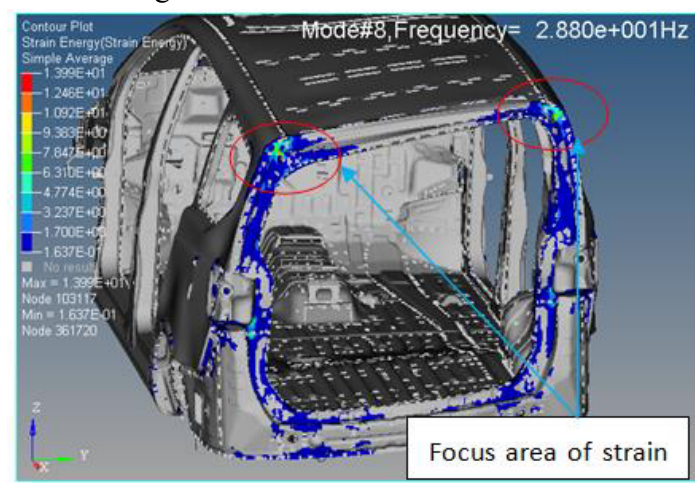

Figure 7. Strain energy distribution vector

The pillar $\mathrm{D}$ is the position that has the largest strain energy, and the distribution is an extreme focus. It shows that the stiffness of pillar D is very weak, so the tail torsion will be improved by optimizing this area

\subsection{Mode optimization analysis}

The pillar D is the weak stiffness area that results in the lower tail mode from the above discussion. Now, this area is optimized and the schemes are shown in Figure 8 and Figure 9.

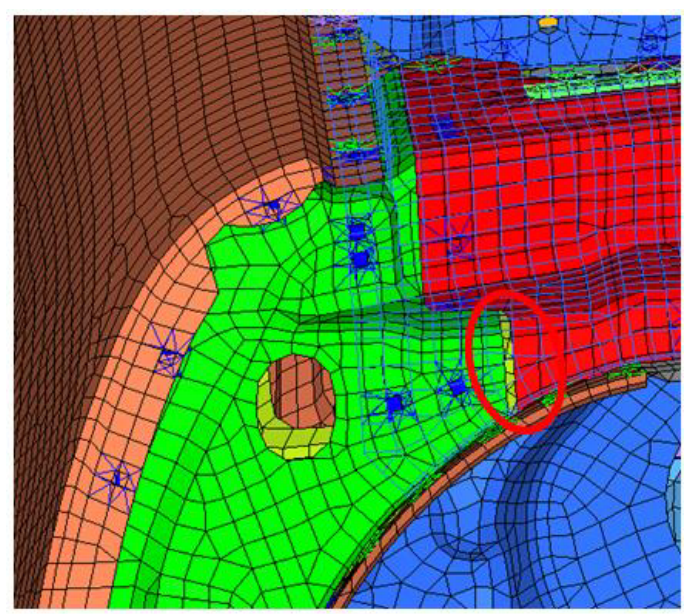

Figure 8. Old structure

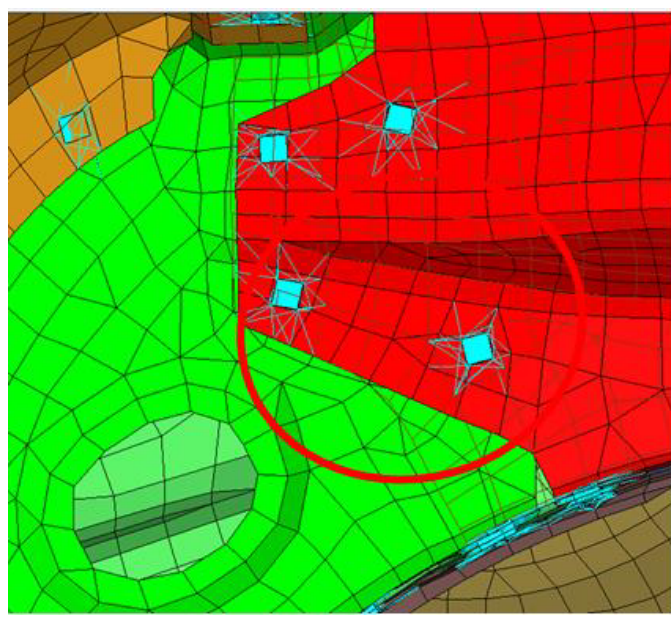

Figure 9. Scheme 1: strengthening the overlap joint

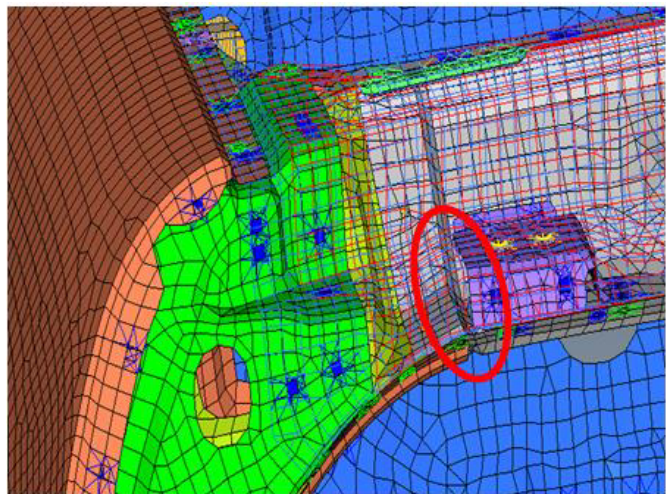

Figure 10. Old structure 


\section{ICETA 2015}

The overlap joint between red panel and green panel has the large gap from Figure 9. So the red panel is improved by strengthening that area from scheme 1 .

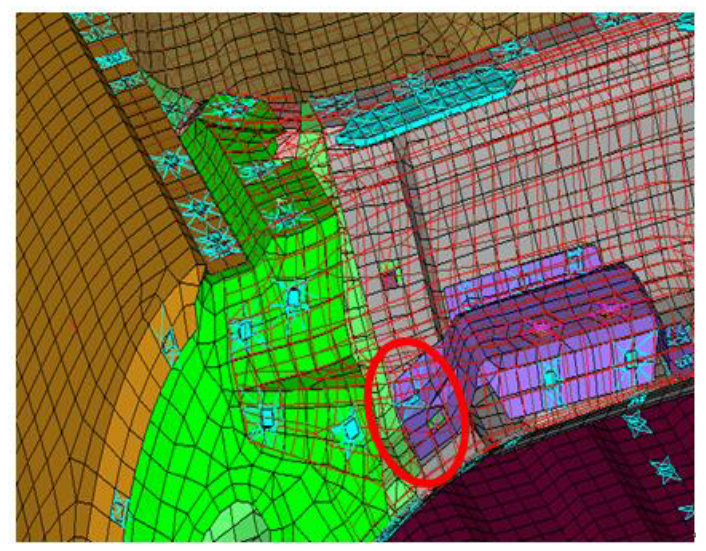

Figure 11. Scheme 2: prolonging the hinge strengthening panel

Prolonging the hinge and strengthening panel makes the area stiffness more continuous, and the stiffness of this area is improved a lot. It can be seen from scheme 2 .

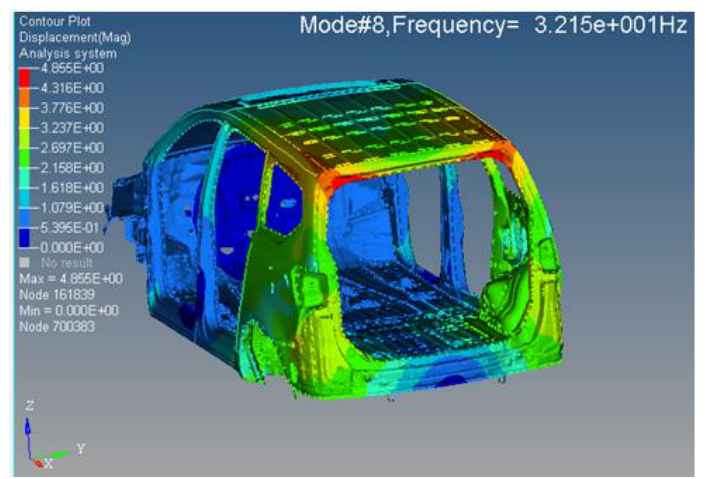

Figure 12. Optimized mode result

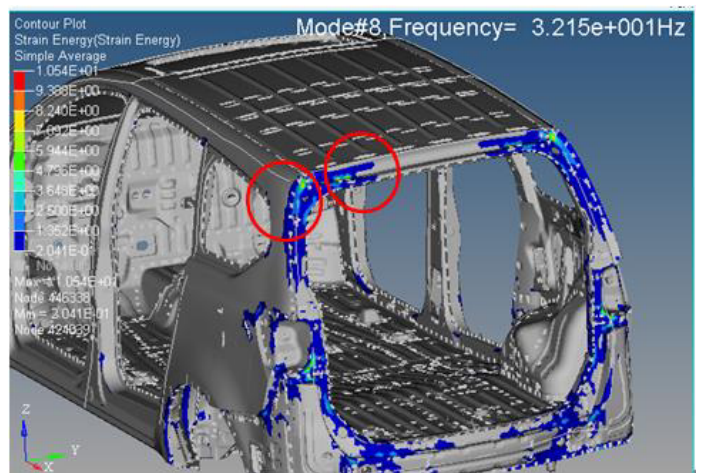

Figure 13. Optimized mode strain energy
The tail mode is improved from $28.80 \mathrm{~Hz}$ to 32.15 $\mathrm{Hz}$ after optimizing, which meets the requirement of $32 \mathrm{~Hz}$. And the strain energy distribution is largely improved. That can be seen in the Figure 6. The strain focus is effectively dispersed so the stiffness of pillar $\mathrm{D}$ is obviously improved, and the tail mode is increased from $28.80 \mathrm{~Hz}$ to $32.15 \mathrm{~Hz}$.

\section{CONCLUSIONS}

This paper takes the BIW model as the research object, and completes mode analysis. Utilizing the strain energy method to optimize, the conclusions are drawn as follows:

1) It's verified that the finite element model is correct by comparing the simulation results with the test results. Utilizing this model to optimize further, so the optimization result is believable and correct.

2) The weak stiffness of pillar D is the reason why the mode is low, which is attained by strain energy method.

3) The strain focus is effectively dispersed so the stiffness of pillar D is obviously improved, and the tail mode is increased from $28.80 \mathrm{~Hz}$ to $32.15 \mathrm{~Hz}$. It shows that the strain energy method has an obvious influence on mode optimization.

\section{REFERENCES}

[1] Zhang Guosheng, Meng Fanliang. \& Zhang Tianxia etc.2008. BIW digital modeling and stiffness analysis. Journal of Northeastern University, 29 (2).

[2] MAGOSHI Y, FUJ ISAWA H. \& SUGIURA T1. 2003. Simulation technology applied to vehicle development. Auto Vehicle Technique, 57 (3): 95-1001.

[3] Qu Hao, Wang Xiaohua. \& Tong Zhang. 2010. Mode analysis and optimization of frame body. Highway Traffic Technology. 27 (6): 134-136.

[4] Cheng Changming. \& Xiao Qiang. 2009. BIW mode sensitivity and structure optimization analysis. Journal of Shandong Jiaotong University, 17(2): 2-5.

[5] Meng Fanliang, 2007. The Static and Dynamic Characteristics and Sensitivity Analysis of Car Body, Northeastern University.

[6] Yang Yuekai. 2006. FEM and Static and Dynamic Characteristics of Car Body, Northeastern University.

[7] Xu Li. \& Yi Weijian. 2006. The changed stiffness of concrete frame is achieved by mode strain energy. $\mathrm{Vi}$ bration and Impact, 25(3): 1-51.

[8] HERTING D N1 MSC/ NASTRAN Supper Element Analysis. 1999. New York: Mac NealOSchwendler Corporation. 